\title{
Ubiquitin E3 ligases Atrogin-1 and MuRF1 protein contents are differentially regulated in the rapamycin-sensitive mTOR-S6K1 signaling pathway in $\mathrm{C2C12}$ myotubes
}

Yusuke Nishimura ${ }^{1}$, Ibrahim Musa ${ }^{1}$, Peter Dawson ${ }^{1,2}$, Lars Holm $^{1,2}$, Yu-Chiang Lai ${ }^{1,2,3^{*}}$

${ }^{1}$ School of Sport, Exercise and Rehabilitation Sciences, University of Birmingham,

Birmingham, United Kingdom

${ }^{2}$ MRC Versus Arthritis Centre for Musculoskeletal Ageing Research, University of

Birmingham, Birmingham, United Kingdom

${ }^{3}$ Institute of Metabolism and Systems Research, University of Birmingham, Birmingham, United Kingdom

Running Title: mTORC1/S6K1 and ubiquitin E3 ligase protein content

*Address for correspondence:

Dr. Yu-Chiang Lai, Ph.D.

School of Sport, Exercise and Rehabilitation Sciences

University of Birmingham, Edgbaston, Birmingham, B15 2TT, UK

Phone: +44 (0)121 4145315

Email: y.lai.1@bham.ac.uk 


\section{Abstract}

2 Muscle-specific ubiquitin E3 ligases, Atrogin-1 and MuRF1, are highly expressed in multiple

3 conditions of skeletal muscle atrophy. The PI3K/Akt/FoxO signaling pathway is well known

4 to regulate Atrogin-1 and MuRF1 gene expressions. Evidence supporting this is largely based

5 on stimuli by insulin and IGF-1, that activate anabolic signaling, including Akt and Akt-

6 dependent transcription factors. However, Akt activation also activates the mammalian target

7 of rapamycin complex 1 (mTORC1) which induces skeletal muscle hypertrophy. However,

8 whether mTORC1-dependent signaling has a role in regulating Atrogin-1 and/or MuRF1

9 gene and protein expression is currently unclear. In this study, we confirmed that activation

10 of insulin-mediated Akt signaling suppresses both Atrogin-1 and MuRF1 protein content and

11 that inhibition of Akt increases both Atrogin-1 and MuRF1 protein content in C2C12

12 myotubes. Interestingly, inhibition of mTORC1 using a specific mTORC1 inhibitor,

13 rapamycin, increased Atrogin-1, but not MuRF1, protein content. Furthermore, activation of

14 AMP-activated protein kinase (AMPK), a negative regulator of the mTORC1 signaling

15 pathway, also showed distinct time-dependent changes between Atrogin-1 and MuRF1

16 protein content, suggesting differential regulatory mechanisms between Atrogin-1 and

17 MuRF1 protein content. To further explore the downstream of mTORC1 signaling, we

18 employed a specific S6K1 inhibitor, PF-4708671, and found that Atrogin-1 protein content

19 was dose-dependently increased with PF-4708671 treatment, whereas MuRF1 protein content

20 was not significantly altered. Overall, our results indicate that Atrogin-1 and MuRF1 protein

21 contents are regulated by different mechanisms, the downstream of Akt, and that Atrogin-1

22 protein content can be regulated by rapamycin-sensitive mTOR-S6K1 dependent signaling

23 pathway.

25 KEY WORDS: The ubiquitin proteasome system, skeletal muscle 


\section{Abbreviations:}

27 MuRF1, Muscle-specific RING finger protein 1

28 MAFbx, Muscle atrophy F-box protein

29 PI3K, phosphoinositide 3-kinase

30 FoxO, forkhead box

31 mTORC, mechanistic target of rapamycin complex

32 TSC2, tuberous sclerosis complex 2

33 S6K, p70 ribosomal S6 kinase

34 AMPK, adenosine monophosphate activated protein kinase

35 SDS-PAGE, sodium dodecyl sulfate- polyacrylamide gel electrophoresis

36 PVDF, polyvinylidene fluoride

37 TBS-T, Tris-buffered saline Tween-20

38 ANOVA, analysis of variance

39 CON, control

40 


\section{1. Introduction}

42 Atrogin-1 (also known as Muscle atrophy F-box protein: MAFbx or FBXO32) and Muscle-

43 specific RING finger protein 1 (MuRF1 or TRIM63) are muscle specific E3 ligases and their

44 expression is highly associated with various skeletal muscle atrophic models [1, 2]. In

45 agreement with above, a plethora of studies have confirmed that Atrogin-1 and MuRF1

46 mRNA expression are useful molecular biomarkers of skeletal muscle atrophy [3]. Although

47 both Atrogin-1 and MuRF1 gene expressions increase in almost all atrophic models, various

48 muscle atrophic conditions (e.g., fasting, immobilization, diabetes, insulin resistance) are

49 likely to alter multiple signaling pathways to control Atrogin-1 and MuRF1 gene and protein

50 expression [4]. While the PI3K-Akt signaling pathway is known to regulate Atrogin-1 and

51 MuRF1 gene expression, the mechanisms that regulate protein content of these two E3

52 ligases remain to be elucidated. Many studies have assumed that mRNA expressions

53 implicitly reflect the corresponding changes of protein content, but in reality the expression

54 levels of individual mRNA and its corresponding protein are indeed poorly correlated $[5,6]$.

55 The poor correlation can be explained by multiple processes, including transcription and

56 degradation of mRNAs, translation, folding, and degradation of proteins [7, 8]. As protein is

57 the final product executing gene function, direct measurement of protein content should be

58 more relevant to biological functions [8, 9]. However, in the cases of Atrogin-1 and MuRF1,

59 poor quality of antibodies is often a major obstacle to reveal protein content in biological

60 samples $[3,10,11]$.

$62 \mathrm{PI} 3 \mathrm{~K} /$ Akt/forkhead box (FoxO) signaling is one of the most well studied pathways known to

63 regulate Atrogin-1 and MuRF1 mRNA transcription expression [12-14]. Studies have shown

64 that treatment of IGF-1 or an introduction of constitutively active Akt prevents both Atrogin-

651 and MuRF1 mRNA transcription expression in C2C12 myotubes [12, 13]. In addition, 
66 denervation-induced skeletal muscle atrophy was prevented by IGF-1 treatment [13]. IGF-1

67 increases Akt phosphorylation and suppresses Atrogin-1 and MuRF1 mRNA transcription

68 expression in mouse skeletal muscle [13], indicating a link between Akt and Atrogin-

69 1/MuRF1 axis in skeletal muscle atrophy. Mechanistically, Akt phosphorylates the

70 transcription factor FoxO to induce FoxO nuclear exclusion, which downregulates FoxO-

71 dependent gene transcription [15]. A study has also confirmed that overexpression of FoxO3a

72 in mouse skeletal muscle is able to induce Atrogin-1 mRNA expression and an atrophic

73 phenotype [12]. In contrast, siRNA knockdown of FoxO1-3 inhibits Atrogin-1 promoter

74 activity measured by Atrogin-1 luciferase reporter constructs during fasting-induced muscle

75 atrophy [12]. All these findings have evidenced that Akt-FoxO axis is critical for regulating

76 Atrogin-1 and MuRF1 mRNA transcriptional expression. However, some contradictory

77 results have also been reported. For example, a study showed that deletion of Akt1 or Akt2

78 did not alter Atrogin-1 mRNA and protein expressions in mouse skeletal muscle [16].

79 Atrogin-1 and MuRF1 mRNA expression, including Atrogin-1 protein content, were shown

80 to be unchanged in ageing-induced muscle atrophy, where Akt activity and FoxO3a

81 phosphorylation were elevated, compared to young control skeletal muscles [17]. These

82 contradictory findings raise the question of whether Akt-FoxO axis is the solely signaling

83 pathway that regulate Atrogin-1 and MuRF1 expression.

mTORC1 plays an important role in regulating protein synthesis and autophagy-lysosome

86 system [18], and its activation has been well associated with skeletal muscle hypertrophy [19,

87 20]. Surprisingly, the involvement of mTORC1 in regulating muscle protein degradation has

88 not been well investigated. A recent study led by Zhao et al. [21] suggested that mTOR

89 (including mTORC1 and mTORC2) may be involved in the regulation of protein degradation

90 in $\mathrm{C} 2 \mathrm{C} 12$ myotubes. Their previous study has shown that treatment of rapamycin, a specific 
91 mTORC1 inhibitor, can increase protein degradation in C2C12 myotubes [22], which lead

92 the authors to suggest mTORC1 as a contributor for controlling protein degradation.

93 Furthermore, there is also evidence suggesting that Atrogin-1 and MuRF1 mRNA

94 expressions are regulated by distinct signaling mechanisms. Sacheck et al. [22] showed that

95 rapamycin treatment increases Atrogin-1, but not MuRF1, mRNA expression. However,

96 proof at protein level is currently lacking and such information is needed to better understand

97 what the signaling mechanisms are controlling Atrogin-1 and MuRF1 protein content, which

98 essentially execute the enzymatic ubiquitin E3 ligase activity.

100 The present study therefore aims to investigate whether the downstream of Akt, such as

101 mTORC1 and S6K1 signaling pathway, is involved in controlling Atrogin-1 and MuRF1

102 protein content in C2C12 myotubes. Using small molecules inhibiting mTORC1 or S6K1

103 activity, we demonstrated that Atrogin-1, but not MuRF1, protein content is regulated in the

104 rapamycin-sensitive mTOR and S6K1-dependent signaling pathways. Our results suggest

105 that the role of Akt-FoxO is not the only signaling pathway regulating Atrogin-1 protein

106 content and that the downstream of Akt, such as the rapamycin-sensitive mTOR and S6K1-

107 dependent signaling pathways, are involved in regulating Atrogin-1 protein content in

108 skeletal muscle.

110 2. MATERIALS AND METHODS

1112.1 C2C12 cell culture

112 Mouse skeletal muscle C2C12 myoblast cells were obtained from the American Type Culture

113 Collection (ATCC, Manassas, VA, USA). Cells were seeded and maintained in Dulbecco's

114 Modified Eagle Medium (DMEM; Thermo Fisher Scientific, Loughborough, UK, 31966021)

115 containing GlutaMAX, $25 \mathrm{mM}$ of glucose, and $1 \mathrm{mM}$ of sodium pyruvate, supplemented with 
$11610 \%(\mathrm{v} / \mathrm{v})$ of Hyclone fetal bovine serum (FBS, Fisher Scientific, Loughborough, UK,

117 SV30180.03), 1\% (v/v) of Penicillin-Streptomycin (10 000 Units/mL-ug/mL, Thermo Fisher

118 Scientific, Loughborough, UK, 15140122). Myoblasts were seeded onto six-well multidishes

119 (greiner bio-one, 657 160) and when confluency reached at 90\%, myoblasts were

120 differentiated into myotubes in DMEM supplemented with 2\% (v/v) of horse serum (Sigma-

121 Aldrich, Cambridgeshire, UK, H1270), 1\% (v/v) of Penicillin-Streptomycin. The media was

122 changed every $48 \mathrm{~h}$. Cultures were maintained in a humified incubator at $37 \square$ with an

123 atmosphere of $5 \%$ of $\mathrm{CO} 2$ and $95 \%$ of air.

\subsection{Drug reconstitution and cell treatment}

126 Akt1/2/3 inhibitor MK-2206 dihydrochloride (ApexBio, A3010), Rapamycin (Sigma-Aldrich,

127 553211), adenosine monophosphate (AMP)-activated protein kinase (AMPK) activator 991

128 (AOBIOUS, MA, USA, AOB8150), S6K1 Inhibitor, PF-4708671 (Sigma-Aldrich, Dorset,

129 UK, 559278) were prepared in DMSO and treatment conditions were described in the figure

130 legend. Insulin solution human was obtained from Sigma (Sigma Aldrich, Dorset, UK,

131 I9278).

134 Cells were lysed in ice-cold sucrose lysis buffer containing: $250 \mathrm{mM}$ of sucrose, $50 \mathrm{mM}$ of

135 Tris-base (pH 7.5), $50 \mathrm{mM}$ of sodium fluoride, $10 \mathrm{mM}$ of sodium $\beta$-glycerophosphate, $5 \mathrm{mM}$

136 of sodium pyrophosphate, $1 \mathrm{mM}$ of EDTA, $1 \mathrm{mM}$ of EGTA, $1 \mathrm{mM}$ of benzamidine, $1 \mathrm{mM}$ of

137 sodium orthovanadate, $1 \times$ complete Mini EDTA-free protease inhibitor cocktail (Roche), $1 \%$

138 of Triton X-100, and $100 \mathrm{mM}$ of 2-chloroacetamide. Cell lysates were centrifuged for 15

139 minutes at $13000 \mathrm{~g}$ at $4^{\circ} \mathrm{C}$ and the supernatant was stored at $-80^{\circ} \mathrm{C}$ before analysis for total

140 protein concentrations using the Bradford protein assay (Thermo Fisher Scientific, 
141 Leicestershire, UK, 23200). Protein in each sample was quantified from a standard curve

142 using BSA standards (Thermo Fisher Scientific, Leicestershire, UK, 23209).

\subsection{Western blot}

145 Cell lysates were prepared in 1x NuPAGE LDS sample buffer (Invitrogen, NP0008)

146 containing 2-mercaptoethanol (final concentration 1.5\%) and left to denature overnight at

147 room temperature. Prepared cell lysates (10-15 $\mu \mathrm{g}$ of total protein) were loaded into $8 \%$ or

$14810 \% \mathrm{Bis} /$ Tris gels prior to sodium dodecyl sulfate- polyacrylamide gel electrophoresis (SDS-

149 PAGE). Gels were run in 1x MOPS buffer for approximately 60 minutes at 140V. Proteins

150 were transferred onto $0.2 \mu \mathrm{m}$ polyvinylidene fluoride (PVDF) membranes (Millipore,

151 Hertfordshire, UK) for 1 hour at 100V. Membranes were blocked in 5\% of milk diluted in

152 Tris-buffered saline Tween-20 (TBS-T): $137 \mathrm{mM}$ of sodium chloride, $20 \mathrm{mM}$ of Tris-base

$1537.5 \mathrm{pH}, 0.1 \%$ of Tween-20 for 1 hour. After blocking, membranes were washed 3 times for 5

$154 \mathrm{~min}$ in TBS-T before being incubated overnight at $4^{\circ} \mathrm{C}$ with the appropriate primary

155 antibodies (Table 1). Membranes were washed 3 times for $5 \mathrm{~min}$ in TBS-T prior to

156 incubation in horse radish peroxidase-conjugated secondary antibodies (see Supplementary

157 Table 1) at room temperature for $1 \mathrm{~h}$. Membranes were washed a further three times in TBS-

158 T prior to antibody detection using enhanced chemiluminescence horseradish peroxidase

159 substrate detection kit (Millipore, Hertfordshire, UK). Imaging was undertaken using a

160 G:BOX Chemi-XR5 (Syngene, Cambridgeshire, UK). Band intensities were quantified using

161 ImageJ/Fiji (NIH, Bethesda, MD, USA). Phosphorylation levels were determined by the

162 expression of phosphorylated protein divided by expression of non-phosphorylated total

163 protein. Vinculin was used as the loading control. 


\subsection{Statistical analysis}

166 The statistical analyses were performed using Prism version 8.1.2 (GraphPad Software, San

167 Diego, California USA, www.graphpad.com). Values of $P<0.05(*)$ were considered

168 statistically significant. For time course and dose-response experiments, a one-way analysis

169 of variance (ANOVA) was performed with Dunnett's post-hoc test compared to control

170 (CON). Data are presented as mean \pm SD. All experiments were performed in duplication and 171 repeated at least twice.

\section{3. Results}

1733.1 Evidence of Insulin/Akt/FoxO signaling pathway modulating Atrogin-1 and MuRF1

174 protein content

175 We first confirmed if insulin/Akt/FoxO signaling pathway is sufficient to modulate both

176 Atrogin-1 and MuRF1 protein content in C2C12 myotubes. Using an allosteric Akt inhibitor

177 (MK-2206), we showed that Atrogin-1 protein content was significantly increased at $3 \mathrm{~h}, 6 \mathrm{~h}$,

178 and $9 \mathrm{~h}$ after the treatment of $10 \mu \mathrm{M}$ MK-2206 (Fig. $1 \mathrm{~B}$ ). MuRF1 protein content was also

179 significantly increased at $6 \mathrm{~h}$ after the treatment of MK-2206 (Fig. 1 C). In line with a

180 previous study [23], Akt phosphorylation at $\mathrm{Ser}^{473}$ and $\mathrm{Thr}^{308}$ was completely abolished over

181 the course of $9 \mathrm{~h}$ treatment with MK-2206 (Fig. 1 A). We also confirmed that inhibition of

182 Akt activity prevents FoxO1 and FoxO3a phosphorylation and reduces S6K1 and rpS6

183 phosphorylation (Fig. 1 A).

185 Atrogin-1 protein content was significantly decreased at $3 \mathrm{~h}, 6 \mathrm{~h}$, and $9 \mathrm{~h}$ following the

186 treatment of $100 \mathrm{nM}$ insulin stimulation (Fig. 1 B). MuRF1 protein content was also

187 significantly decreased at $6 \mathrm{~h}$ and $9 \mathrm{~h}$ after insulin treatment (Fig. $1 \mathrm{C}$ ). As expected, insulin

188 stimulated Akt phosphorylation at both $\operatorname{Ser}^{473}$ and $\mathrm{Thr}^{308}$ sites. The enhanced Akt activity was 
189 also confirmed by the increases of its downstream, such as FoxO1, FoxO3a, S6K1, and rpS6

190 phosphorylation (Fig. 1 A).

191

3.2 Atrogin-1, but not MuRF1, protein content is increased by the rapamycin sensitive

mTORC1 inhibition

194 Treatment with Rapamycin can specifically inhibit mTORC1 activity without directly

195 affecting mTORC2 activity, but a long-term treatment ( $\geq 24 \mathrm{~h})$ is known to inhibit mTORC2

196 activity [24]. Therefore, we have limited the treatment time of small molecules to not more

197 than $9 \mathrm{~h}$. Interestingly, Atrogin-1 protein content was increased at $3 \mathrm{~h}, 6 \mathrm{~h}$, and $9 \mathrm{~h}$ following

198 the treatment of $100 \mathrm{nM}$ rapamycin (Fig. 2 B). Despite that Atrogin-1 protein content was

199 increased, MuRF1 protein content remained unchanged (Fig. 2 C). As anticipated, rapamycin

200 treatment completely inhibited S6K1 and rpS6 phosphorylation (Fig. 2 A) without inducing a

201 significant change in Akt phosphorylation $(P=0.38)$.

202

3.3 Distinct time-dependent changes of Atrogin-1 and MuRF1 protein content following

\section{AMPK activation}

205 AMPK activation is known to inhibit mTORC1 activity [25] via the phosphorylation of

206 tuberous sclerosis complex 2 (TSC2) [26] and Raptor [27]. To further investigate the role of

207 mTORC1 on the regulation of Atrogin-1 and MuRF1 protein content, we used a direct

208 AMPK activator, 991, to increase AMPK activity in C2C12 myotubes $[25,28]$. Interestingly,

209 Atrogin-1 protein content was increased rapidly at $3 \mathrm{~h}$ and $6 \mathrm{~h}$, despite returning to the basal

210 level after $9 \mathrm{~h}$ of treatment (Fig. $3 \mathrm{~B}$ ). In contrast, MuRF1 protein content had obviously

211 delayed increases at $6 \mathrm{~h}$ and $9 \mathrm{~h}$ after 991 treatment (Fig. $3 \mathrm{C}$ ). These results again suggest

212 that Atrogin-1 and MuRF1 protein content are regulated by distinct signaling mechanisms.

213 As expected, ULK1 phosphorylation at $\operatorname{Ser}^{555}$ was increased by the treatment of 991 (Fig. 3 
214 A) [29] and the inhibition of mTORC1 activity was confirmed by showing a decrease in

215 S6K1 and rpS6 phosphorylation (Fig. 3 A).

\subsection{Atrogin-1 protein content is increased by S6K1 inhibition}

218 To further explore the distinct mechanisms that regulate Atrogin-1 and MuRF1 protein

219 content, we asked whether mTORC1 downstream, such as S6K1, is involved in regulating

220 Atrogin-1 or MuRF1 protein content. Using a specific S6K1 inhibitor [30], we showed that

221 Atrogin-1 (Fig. 4 B) protein content was increased in a dose-response manner, where

222 significant increases was seen with the treatment of $40 \mu \mathrm{M}$ and $50 \mu \mathrm{M}$ PF-4708671. Instead

223 of increasing, MuRF1 protein content was indeed decreased at $50 \mu \mathrm{M}$ (Fig. 4 C). Inhibition

224 of S6K1 was confirmed by the observation of reduced rpS6 phosphorylation (Fig. 4 A). As

225 expected, the phosphorylation of S6K1 was increased by the treatment of PF-4708671 [30]

226 (Fig. 4 A). Next, we performed Pearson's correlation coefficient to identify the relationship

(Fig. 4 D). Interestingly, a strong negative correlation was observed between p-

$\mathrm{rpS}^{\mathrm{Ser} 240 / 244} / \mathrm{rpS6}$ and Atrogin-1 $(\mathrm{r}=-0.90, P<0.0001)$, whereas no significant association

was observed between p-rpS6 ${ }^{\text {Ser240/244 } / r p S 6}$ and MuRF1 $(r=0.17, P=0.44)$.

232 To confirm that S6K1 inhibition increases Atrogin-1, but not MuRF1, protein content, we

233 performed a time course experiment using $30 \mu \mathrm{M}$ of PF-4708671 for up to $24 \mathrm{~h}$ (Fig. 5 A).

234 As anticipated, the protein content of Atrogin-1 was increased over the course of PF-4708671

235 treatment at 3 h, 6 h, and 24 h (Fig. 5 B). Although MuRF1 protein content (Fig. 5 C)

236 remained unchanged over majority of the time points, there was still an unexpected increase

237 occurred at $6 \mathrm{~h}$ after PF-4708671 treatment. 


\section{4. Discussion}

240 The gene expression of Atrogin-1 and MuRF1 are highly associated with almost all kinds of

241 skeletal muscle atrophy [1-3]. Genetic studies have also shown that knockout of Atrogin-1 or

242 MuRF1 partially rescue denervation-induced skeletal muscle atrophy [1]. However, the

243 molecular mechanisms of how Atrogin-1 and MuRF1 contribute to skeletal muscle atrophy

244 are still unclear. The most recent study has indicated that the enzymatic activity of these

245 ubiquitin E3 ligases is particularly important in controlling skeletal muscle mass [31].

246 Therefore, obtaining information relevant to the regulation of Atrogin-1 and MuRF1 protein

247 content will provide an alternative opportunity to manipulate their functional E3 ligase

248 activity. This information will also help identify new therapeutic targets to treat and/or

249 prevent skeletal muscle atrophy. Here, we have made use of small molecules to evaluate

250 some key signaling pathways that modulate Atrogin-1 and MuRF1 protein contents in C2C12

251 myotubes. In accordance with previous studies, we confirmed that insulin/Akt/FoxO pathway

252 is sufficient to modulate both Atrogin-1 and MuRF1 protein contents, which is in agreement

253 with the tendency of measuring mRNA transcriptional expression [12-14, 22]. Further

254 investigation revealed that Atrogin-1, but not MuRF1, protein content is predominantly

255 increased when rapamycin-sensitive signaling pathways is inhibited. These findings show

256 that Atrogin-1 and MuRF1 protein contents are regulated through different mechanisms

257 downstream of Akt. More interestingly, our studies also revealed that Atrogin-1 protein

258 content can be regulated by S6K1 dependent signaling pathway.

260 Inactivation of PI3K/Akt/FoxO signaling pathway is well known as an "atrophic signal" that

261 increases both MuRF1 and Atrogin-1 mRNA expression [32]. However, few studies have

262 investigated whether MuRF1 and Atrogin-1 protein contents are regulated in accordance with

263 their gene/mRNA expressions. The current study confirmed that protein content of both 
264 Atrogin-1 and MuRF1 were suppressed by insulin, whereas Atrogin-1 and MuRF1 protein

265 contents were upregulated by the treatment of MK-2206. These findings are consistent with

266 the mRNA expressions investigated by previous studies [12-14, 22].

268 In the present study, we showed that Atrogin-1 protein content was increased after $3 \mathrm{~h}$

269 treatment of rapamycin, whereas MuRF1 protein content was not changed throughout the

270 time course (Fig. 2). This data indicates that inhibition of mTORC1 signaling can enhance

271 Atrogin-1, but not MuRF1, protein content. This is indeed consistent with a previous study

272 reported that inhibition of rapamycin-sensitive signaling pathway increases Atrogin-1, but not

273 MuRF1, mRNA expression [22]. Our findings strengthened the previous evidence of mRNA

274 data [22] by showing that inhibition of rapamycin-sensitive mTOR-S6K1 signaling pathway

275 also induces an increase in Atrogin-1 protein content.

277 The most interesting findings in the present study are that Atrogin-1 and MuRF1 protein

278 contents can be regulated differently, and that Atrogin-1 protein content is regulated by

279 rapamycin-sensitive and S6K1 dependent signaling pathways. In the present study, the

280 phosphorylation of FoxO3a at $\mathrm{Thr}^{32}$ and FoxO1 at $\mathrm{Thr}^{24}$ was not altered after rapamycin or

281 PF-4708671 treatment, suggesting that FoxOs are not the most critical factor regulating

282 Atrogin-1 (as well as MuRF1) protein content. Multiple transcription factors, including the

$283 \mathrm{NF}-\kappa \mathrm{B}$ transcription factors CCAAT/enhancer-binding protein- $\beta(\mathrm{C} / \mathrm{EBP} \beta)$ and Smad3, can

284 work cooperatively to regulate Atrogin-1 mRNA transcription Atrogin-1 [3,33]. Thus,

285 complex cooperative mechanisms of transcription factors might have been involved in the

286 distinct protein expression patterns between Atrogin-1 and MuRF1 protein content. In

287 supporting our finding that Atrogin-1 protein content is regulated by S6K1 dependent

288 signaling, previous studies have also shown that the absence of S6K1 causes skeletal muscle 
289 atrophy in mice [34]. In addition, Marabita et al. [35] reported that S6K1 is required for the

290 prevention of protein aggregation during skeletal muscle hypertrophy in mice. These

291 observations led us to hypothesize that protein quality control, mainly protein degradation, is

292 the mechanism inducing the increased Atrogin-1 protein content, when rapamycin-sensitive

293 mTOR-S6K1 signaling is inhibited. However, future studies should confirm this hypothesis

294 by investigating the process of Atrogin-1 protein turnover rate, and subsequent protein

295 content.

297 The mTORC1 signaling pathway has been shown as a positive regulator of skeletal muscle

298 mass in several models of hypertrophy [19, 20, 36]. In support of age-related muscle loss,

299 studies have demonstrated that muscle contraction-induced activation of mTORC1 signaling

300 is impaired with ageing $[37,38]$. In contrast, constant activation of mTORC1 is known to

301 cause myopathy, but not hypertrophy [39]. Moreover, a most recent study led by Joseph et al.

302 [40] showed that mTORC1 signaling pathway is indeed hyperactivated in age-related muscle

303 loss with a concomitant increase in both Atrogin-1 and MuRF1 mRNA expression in basal

304 rat skeletal muscle. More interestingly, a partial inhibition of mTORC1 via RAD001 restored

305 age-related skeletal muscle loss [40]. RAD001 treatment also decreased MuRF1 mRNA

306 expression while Atrogin-1 mRNA was not altered in ageing muscle. We cannot directly

307 compare our findings to their results as they did not report Akt activity and information of

308 MuRF1 and Atrogin-1 protein contents was not available. Nonetheless, these findings

309 indicate the importance of fine tuning the mTORC1 activity in maintaining skeletal muscle

310 mass and Atrogin-1 and/or MuRF1 may be responsible for this.

312 Protein content is determined by protein turnover, which is a continuous process of protein

313 synthesis and protein degradation [41]. In this study, Atrogin-1 and MuRF1 protein contents 
314 were investigated following time-course and/or dose-dependent small molecule treatments,

315 which is a snapshot in time of the impact of the protein turnover kinetics on protein balance.

316 mTORC1 is a well-known signaling pathway to control protein synthesis. Thus, after the

317 treatment of rapamycin or PF-4708671, a decrease in protein synthesis would be expected

318 and a greater decrease in protein degradation would, in theory, contribute to the observed

319 increase in Atrogin-1 protein content. Post-translational modifications and the subsequent

320 degradation make it more complicated to understand how protein content is regulated. For

321 example, many ubiquitin E3 ligases have been implicated to regulate their own protein

322 abundance [42] because most of E3 ligases have the ability to ubiquitylate itself (known as

323 autoubiquitylation) and trigger self-degradation processes (either via proteasome or

324 autophagic lysosome). For example, the greater autoubiquitylation usually demonstrates

325 greater E3 ligase activity [11], which was observed in MuRF1 via in vitro reaction [1].

326 However, the degree of autoubiquitylation on MuRF1 and Atrogin-1 is currently not clear in

327 any of muscle atrophy conditions. Although it is not clear from the present study,

328 autoubiquitylation might have been involved in the regulation of Atrogin-1 and MuRF1

329 protein contents.

331 While our findings suggest that Atrogin-1 and MuRF1 protein contents are regulated by

332 different signaling mechanisms, future studies should determine which molecules in the

333 rapamycin-sensitive mTOR-S6K1 signaling cascade are responsible for regulating Atrogin-1

334 protein content. With the use of our protein content data, other studies should also investigate

335 whether E3 ligase activity of MuRF1 and/or Atrogin-1 is associated with their protein content,

336 and thus a measurement of protein content can be used as a biomarker for E3 ligase activity

337 or vice versa. Additionally, protein degradation contributes half of the equation to determine

338 protein content (i.e., protein synthesis - protein degradation = protein content). Thus, 
339 determining degradation mechanisms of Atrogin-1 and MuRF1 protein contents is also

340 important to modulate protein half-life. Thus, understanding of the degradation mechanisms

341 of Atrogin-1 and MuRF1 is required as an important step towards understanding the

342 underlying mechanisms of skeletal muscle atrophy and manipulating their functional E3

343 ligase activity.

\section{5. Conclusions}

345 Based on the findings from the preset study and the existing literature, we propose potential

346 signaling mechanisms that may be involved in the regulation of Atrogin-1 and MuRF1

347 protein contents in skeletal muscle (Fig. 6). The anabolic Akt signaling, which can be

348 activated by Insulin/IGF-1, is a critical upstream signal to modulate MuRF1 and Atrogin-1 at

349 both gene and protein expression levels. However, Atrogin-1, but not MuRF1, protein

350 content is increased when the rapamycin-sensitive and S6K1 dependent signaling pathways

351 are inhibited. Thus, the regulatory mechanisms of protein content are distinct between

352 Atrogin-1 and MuRF1. Our study provides evidence that Atrogin-1 protein content can be

353 regulated by the rapamycin-sensitive mTOR-S6K dependent signaling pathway. Future

354 studies should determine the underlying mechanisms by which the rapamycin-sensitive

355 mTOR-S6K1 signaling regulates Atrogin-1 protein content. 


\section{ACKNOWLEDGMENT}

358 Fig. 6 was created with BioRender.com.

\section{FUNDING SUPPORT}

360 Y.L. was supported by MRC Versus Arthritis Centre for Musculoskeletal Ageing Research

361 (MR/P021220/1). Y.N. was supported by the Postgraduate Research Scholarship Fund at the

362 University of Birmingham. I.M. was supported by Tertiary Education Trust Fund overseas

363 scholarship award (TETF/ES/UNIV/KOGI/ASTD/2018). P.D. was supported by MRC

364 Versus Arthritis Centre for Musculoskeletal Ageing Research.

\section{CRediT authorship contribution statement}

366 Yusuke Nishimura: Conceptualization, Investigation, Visualization, Writing - original draft,

367 Writing - review \& editing. Ibrahim Musa: Investigation, Writing - review \& editing. Peter

368 Dawson: Writing - review \& editing. Lars Holm: Writing - review \& editing. Yu-Chiang Lai:

369 Conceptualization, Writing - review \& editing.

\section{CONFLICT OF INTEREST}

371 The authors have no conflicts of interest to declare. 


\section{References}

374 [1] S.C. Bodine, E. Latres, S. Baumhueter, V.K. Lai, L. Nunez, B.A. Clarke, W.T.

375 Poueymirou, F.J. Panaro, E. Na, K. Dharmarajan, Z.Q. Pan, D.M. Valenzuela, T.M.

376 DeChiara, T.N. Stitt, G.D. Yancopoulos, D.J. Glass, Identification of ubiquitin ligases

377 required for skeletal muscle atrophy, Science (New York, N.Y.) 294(5547) (2001) 1704-8.

378 [2] M.D. Gomes, S.H. Lecker, R.T. Jagoe, A. Navon, A.L. Goldberg, Atrogin-1, a muscle-

379 specific F-box protein highly expressed during muscle atrophy, Proceedings of the National

380 Academy of Sciences 98(25) (2001) 14440-14445.

381 [3] S.C. Bodine, Edward F. Adolph Distinguished Lecture. Skeletal muscle atrophy: Multiple

382 pathways leading to a common outcome, Journal of Applied Physiology 129(2) (2020) 272-

383282.

384 [4] S.C. Bodine, L.M. Baehr, Skeletal muscle atrophy and the E3 ubiquitin ligases MuRF1

385 and MAFbx/atrogin-1, American journal of physiology. Endocrinology and metabolism

386 307(6) (2014) E469-84.

387 [5] B. Schwanhäusser, D. Busse, N. Li, G. Dittmar, J. Schuchhardt, J. Wolf, W. Chen, M.

388 Selbach, Global quantification of mammalian gene expression control, Nature 473(7347)

389 (2011) 337-342.

390 [6] T. Maier, M. Güell, L. Serrano, Correlation of mRNA and protein in complex biological

391 samples, FEBS letters 583(24) (2009) 3966-3973.

392 [7] Y. Liu, A. Beyer, R. Aebersold, On the dependency of cellular protein levels on mRNA

393 abundance, Cell 165(3) (2016) 535-550.

394 [8] C. Buccitelli, M. Selbach, mRNAs, proteins and the emerging principles of gene

395 expression control, Nat Rev Genet 21(10) (2020) 630-644.

396 [9] C. Vogel, E.M. Marcotte, Insights into the regulation of protein abundance from

397 proteomic and transcriptomic analyses, Nature reviews genetics 13(4) (2012) 227-232. 
398 [10] Y. Nishimura, I. Musa, L. Holm, Y.C. Lai, Recent advances in measuring and

399 understanding the regulation of exercise-mediated protein degradation in skeletal muscle,

400 American journal of physiology. Cell physiology (2021).

401 [11] M. Sandri, Protein breakdown in muscle wasting: role of autophagy-lysosome and

402 ubiquitin-proteasome, The international journal of biochemistry \& cell biology 45(10) (2013)

$403 \quad 2121-2129$.

404 [12] M. Sandri, C. Sandri, A. Gilbert, C. Skurk, E. Calabria, A. Picard, K. Walsh, S.

405 Schiaffino, S.H. Lecker, A.L. Goldberg, Foxo transcription factors induce the atrophy-related

406 ubiquitin ligase atrogin-1 and cause skeletal muscle atrophy, Cell 117(3) (2004) 399-412.

407 [13] T.N. Stitt, D. Drujan, B.A. Clarke, F. Panaro, Y. Timofeyva, W.O. Kline, M. Gonzalez,

408 G.D. Yancopoulos, D.J. Glass, The IGF-1/PI3K/Akt pathway prevents expression of muscle

409 atrophy-induced ubiquitin ligases by inhibiting FOXO transcription factors, Molecular cell

$410 \quad 14(3)(2004)$ 395-403.

411 [14] J. Zhao, J.J. Brault, A. Schild, P. Cao, M. Sandri, S. Schiaffino, S.H. Lecker, A.L.

412 Goldberg, FoxO3 coordinately activates protein degradation by the autophagic/lysosomal and

413 proteasomal pathways in atrophying muscle cells, Cell metabolism 6(6) (2007) 472-483.

414 [15] L.P. Van Der Heide, M.F. Hoekman, M.P. Smidt, The ins and outs of FoxO shuttling:

415 mechanisms of FoxO translocation and transcriptional regulation, Biochemical Journal

$416380(2)(2004) 297-309$.

417 [16] T.H. Reynolds 4th, E. Merrell, N. Cinquino, M. Gaugler, L. Ng, Disassociation of

418 insulin action and Akt/FOXO signaling in skeletal muscle of older Akt-deficient mice,

419 American Journal of Physiology-Regulatory, Integrative and Comparative Physiology

$420 \quad 303(11)(2012)$ R1186-R1194. 
421 [17] M. Gaugler, A. Brown, E. Merrell, M. DiSanto-Rose, J.A. Rathmacher, T.H.t. Reynolds,

422 PKB signaling and atrogene expression in skeletal muscle of aged mice, Journal of applied

423 physiology (Bethesda, Md. : 1985) 111(1) (2011) 192-9.

424 [18] R.A. Saxton, D.M. Sabatini, mTOR Signaling in Growth, Metabolism, and Disease, Cell $425169(2)(2017) 361-371$.

426 [19] C.A. Goodman, The Role of mTORC1 in Mechanically-Induced Increases in Translation 427 and Skeletal Muscle Mass, Journal of applied physiology (Bethesda, Md. : 1985) (2019).

428 [20] K. Baar, K. Esser, Phosphorylation of p70(S6 k) correlates with increased skeletal 429 muscle mass following resistance exercise, Am J Physiol 276 (1999).

430 [21] J. Zhao, B. Zhai, S.P. Gygi, A.L. Goldberg, mTOR inhibition activates overall protein 431 degradation by the ubiquitin proteasome system as well as by autophagy, Proceedings of the 432 National Academy of Sciences 112(52) (2015) 15790-15797.

433 [22] J.M. Sacheck, A. Ohtsuka, S.C. McLary, A.L. Goldberg, IGF-I stimulates muscle 434 growth by suppressing protein breakdown and expression of atrophy-related ubiquitin ligases, 435 atrogin-1 and MuRF1, American Journal of Physiology-Endocrinology and Metabolism 436 287(4) (2004) E591-E601.

437 [23] Y.C. Lai, Y. Liu, R. Jacobs, M.H. Rider, A novel PKB/Akt inhibitor, MK-2206, 438 effectively inhibits insulin-stimulated glucose metabolism and protein synthesis in isolated 439 rat skeletal muscle, The Biochemical journal 447(1) (2012) 137-47.

440 [24] D.D. Sarbassov, S.M. Ali, S. Sengupta, J.H. Sheen, P.P. Hsu, A.F. Bagley, A.L.

441 Markhard, D.M. Sabatini, Prolonged rapamycin treatment inhibits mTORC2 assembly and 442 Akt/PKB, Molecular cell 22(2) (2006) 159-68.

443 [25] Y.-C. Lai, S. Kviklyte, D. Vertommen, L. Lantier, M. Foretz, B. Viollet, S. Hallén, M.H. 444 Rider, A small-molecule benzimidazole derivative that potently activates AMPK to increase 
445 glucose transport in skeletal muscle: comparison with effects of contraction and other AMPK

446 activators, Biochemical Journal 460(3) (2014) 363-375.

447 [26] K. Inoki, T. Zhu, K.-L. Guan, TSC2 mediates cellular energy response to control cell

448 growth and survival, Cell 115(5) (2003) 577-590.

449 [27] D.M. Gwinn, D.B. Shackelford, D.F. Egan, M.M. Mihaylova, A. Mery, D.S. Vasquez,

450 B.E. Turk, R.J. Shaw, AMPK phosphorylation of raptor mediates a metabolic checkpoint,

451 Molecular cell 30(2) (2008) 214-226.

452 [28] B. Xiao, M.J. Sanders, D. Carmena, N.J. Bright, L.F. Haire, E. Underwood, B.R. Patel, 453 R.B. Heath, P.A. Walker, S. Hallen, Structural basis of AMPK regulation by small molecule 454 activators, Nature communications 4(1) (2013) 1-10.

455 [29] D.F. Egan, D.B. Shackelford, M.M. Mihaylova, S. Gelino, R.A. Kohnz, W. Mair, D.S.

456 Vasquez, A. Joshi, D.M. Gwinn, R. Taylor, Phosphorylation of ULK1 (hATG1) by AMP-

457 activated protein kinase connects energy sensing to mitophagy, Science (New York, N.Y.)

$458 \quad 331(6016)(2011) 456-461$.

459 [30] L.R. Pearce, G.R. Alton, D.T. Richter, J.C. Kath, L. Lingardo, J. Chapman, C. Hwang,

460 D.R. Alessi, Characterization of PF-4708671, a novel and highly specific inhibitor of p70

461 ribosomal S6 kinase (S6K1), Biochemical Journal 431(2) (2010) 245-255.

462 [31] L.M. Baehr, D.C. Hughes, S.A. Lynch, D. Van Haver, T.M. Maia, A.G. Marshall, L.

463 Radoshevich, F. Impens, D.S. Waddell, S.C. Bodine, Identification of the MuRF1 skeletal

464 muscle ubiquitylome through quantitative proteomics, Function (2021).

465 [32] D.J. Glass, Signalling pathways that mediate skeletal muscle hypertrophy and atrophy,

466 Nat Cell Biol 5(2) (2003) 87-90.

467 [33] L.M. Bollinger, C.A. Witczak, J.A. Houmard, J.J. Brault, SMAD3 augments FoxO3468 induced MuRF-1 promoter activity in a DNA-binding-dependent manner, American journal 469 of physiology-cell physiology 307(3) (2014) C278-C287. 
470 [34] V. Aguilar, S. Alliouachene, A. Sotiropoulos, A. Sobering, Y. Athea, F. Djouadi, S.

471 Miraux, E. Thiaudière, M. Foretz, B. Viollet, P. Diolez, J. Bastin, P. Benit, P. Rustin, D.

472 Carling, M. Sandri, R. Ventura-Clapier, M. Pende, S6 kinase deletion suppresses muscle

473 growth adaptations to nutrient availability by activating AMP kinase, Cell Metab 5(6) (2007)

$474 \quad 476-87$.

475 [35] M. Marabita, M. Baraldo, F. Solagna, J.J.M. Ceelen, R. Sartori, H. Nolte, I. Nemazanyy,

476 S. Pyronnet, M. Kruger, M. Pende, B. Blaauw, S6K1 Is Required for Increasing Skeletal

477 Muscle Force during Hypertrophy, Cell reports 17(2) (2016) 501-513.

478 [36] S.C. Bodine, T.N. Stitt, M. Gonzalez, W.O. Kline, G.L. Stover, R. Bauerlein, E.

479 Zlotchenko, A. Scrimgeour, J.C. Lawrence, D.J. Glass, G.D. Yancopoulos, Akt/mTOR

480 pathway is a crucial regulator of skeletal muscle hypertrophy and can prevent muscle atrophy

481 in vivo, Nat Cell Biol 3(11) (2001) 1014-9.

482 [37] C.S. Fry, M.J. Drummond, E.L. Glynn, J.M. Dickinson, D.M. Gundermann, K.L.

483 Timmerman, D.K. Walker, S. Dhanani, E. Volpi, B.B. Rasmussen, Aging impairs

484 contraction-induced human skeletal muscle mTORC1 signaling and protein synthesis,

$485 \quad$ Skeletal muscle 1(1) (2011) 11.

486 [38] V. Kumar, A. Selby, D. Rankin, R. Patel, P. Atherton, W. Hildebrandt, J. Williams, K.

487 Smith, O. Seynnes, N. Hiscock, M.J. Rennie, Age-related differences in the dose-response

488 relationship of muscle protein synthesis to resistance exercise in young and old men, The

489 Journal of physiology 587(1) (2009) 211-7.

490 [39] P. Castets, S. Lin, N. Rion, S. Di Fulvio, K. Romanino, M. Guridi, S. Frank, L.A.

491 Tintignac, M. Sinnreich, M.A. Rüegg, Sustained activation of mTORC1 in skeletal muscle

492 inhibits constitutive and starvation-induced autophagy and causes a severe, late-onset

493 myopathy, Cell metabolism 17(5) (2013) 731-744. 
494 [40] G.A. Joseph, S.X. Wang, C.E. Jacobs, W. Zhou, G.C. Kimble, W.T. Herman, J.K. Eash,

495 T. Shavlakadze, D.J. Glass, Partial inhibition of mTORC1 in aged rats counteracts the decline

496 in muscle mass and reverses molecular signaling associated with sarcopenia, Molecular and

497 cellular biology 39(19) (2019).

498 [41] J.G. Burniston, Investigating Muscle Protein Turnover on a Protein-by-Protein Basis

499 Using Dynamic Proteome Profiling, Omics Approaches to Understanding Muscle Biology,

500 Springer2019, pp. 171-190.

501 [42] P. De Bie, A. Ciechanover, Ubiquitination of E3 ligases: self-regulation of the ubiquitin

502 system via proteolytic and non-proteolytic mechanisms, Cell Death \& Differentiation 18(9)

503 (2011) 1393-1402.

504

505 


\section{Table 1. Antibodies for western blot}

\begin{tabular}{|c|c|c|c|}
\hline Antibodies & Dilution & Source & Identifier \\
\hline phospho-Ser473 Akt & $1: 1000$ & Cell Signaling Technology & Cat\# 4060 \\
\hline phospho-Thr308 Akt & $1: 1000$ & Cell Signaling Technology & Cat\# 2965 \\
\hline Akt & $1: 1000$ & Cell Signaling Technology & Cat\# 4691 \\
\hline phospho-Thr389 p70 S6 & $1: 1000$ & Cell Signaling Technology & Cat\# 9234 \\
\hline \multicolumn{4}{|l|}{ Kinase } \\
\hline p70 S6 Kinase & $1: 1000$ & Cell Signaling Technology & Cat\# 2708 \\
\hline phospho-Ser240/244 S6 & $1: 8000$ & Cell Signaling Technology & Cat\# 5364 \\
\hline \multicolumn{4}{|l|}{ Ribosomal Protein } \\
\hline S6 Ribosomal Protein & $1: 8000$ & Cell Signaling Technology & Cat\# 2217 \\
\hline phospho-Thr172 AMPK $\alpha$ & $1: 1000$ & Cell Signaling Technology & Cat\# 2535 \\
\hline $\mathrm{AMPK} \alpha$ & $1: 1000$ & Cell Signaling Technology & Cat\# 2532 \\
\hline Atrogin-1 & $1: 1000$ & ECM Biosciences & Cat\# AM3141 \\
\hline MuRF1 & $1: 1000$ & Santa Cruz & Cat\# SC-398608 \\
\hline phospho-Ser555 ULK1 & $1: 1000$ & Cell Signaling Technology & Cat\# 5869 \\
\hline ULK1 & $1: 1000$ & Cell Signaling Technology & Cat\# 4773 \\
\hline Phospho-FoxO1 & $1: 750$ & Cell Signaling Technology & Cat\# 9464 \\
\hline \multicolumn{4}{|l|}{ (Thr24)/FoxO3a (Thr32) } \\
\hline Vinculin & $1: 2000$ & Abcam & Cat\# Ab129002 \\
\hline Anti-mouse IgG, HRP-linked & $1: 10000$ & Cell Signaling Technology & Cat\# 7076 \\
\hline \multicolumn{4}{|l|}{ Antibody } \\
\hline Anti-rabbit IgG, HRP-linked & $1: 10000$ & Cell Signaling Technology & Cat\# 7074 \\
\hline \multicolumn{4}{|l|}{ Antibody } \\
\hline Anti-Rat IgG, HRP-linked & $1: 10000$ & Cell Signaling Technology & Cat\#7077 \\
\hline Antibody & & & \\
\hline
\end{tabular}


508 Figure 1. Insulin/Akt signaling pathway is sufficient to modulate Atrogin-1 and MuRF1

509 protein contents in C2C12 myotubes.

$510 \mathrm{C} 2 \mathrm{C} 12$ myotubes were treated with $\operatorname{DMSO}(0.1 \%, 9 \mathrm{~h})$ as a vehicle control $(\mathrm{CON}), \mathrm{MK} 2206$

$511(10 \mu \mathrm{M})$, or insulin $(100 \mathrm{nM})$ for 3, 6, or 9 h. Lysates were analyzed by SDS-PAGE and

512 western blotting with the indicated antibodies. (A) Representative images from one of two

513 independent experiments. (B) Quantification of Atrogin-1. (C) Quantification of MuRF1.

514 Data are expressed as means $\pm \mathrm{SD}(\mathrm{n}=4)$ fold changes relative to CON. One-way ANOVA

515 with Dunnett's post-hoc test, $* P<0.05, * * P<0.01, * * * P<0.001, * * * * P<0.0001$

516 compared to CON.

517 Figure 2. Rapamycin-sensitive mTOR inhibition increases Atrogin-1, but not MuRF1

518 protein contents in C2C12 myotubes.

$519 \mathrm{C} 2 \mathrm{C} 12$ myotubes were treated with $\operatorname{DMSO}(0.1 \%, 9 \mathrm{~h})$ as a vehicle control $(\mathrm{CON})$ or

520 Rapamycin $(100 \mathrm{nM})$ for 3, 6, or $9 \mathrm{~h}$. Lysates were analyzed by SDS-PAGE and western

521 blotting with the indicated antibodies. (A) Representative images from one of two

522 independent experiments. (B) Quantification of Atrogin-1. (C) Quantification of MuRF1.

523 Data are expressed as means $\pm \mathrm{SD}(\mathrm{n}=4)$ fold changes relative to CON. One-way ANOVA

524 with Dunnett's post-hoc test, $* * * P<0.001$ compared to CON.

525 Figure 3. Inhibition of the mTORC1 pathway by AMPK activator 991 on Atrogin-1 and

526 MuRF1 protein contents in C2C12 myotubes.

$527 \mathrm{C} 2 \mathrm{C} 12$ myotubes were treated with $\operatorname{DMSO}(0.1 \%, 9 \mathrm{~h})$ as a vehicle control $(\mathrm{CON})$ or 991

$528(20 \mu \mathrm{M})$ for 3, 6, or $9 \mathrm{~h}$. Lysates were analyzed by SDS-PAGE and western blotting with the

529 indicated antibodies. (A) Representative images from one of two independent experiments.

530 (B) Quantification of Atrogin-1. (C) Quantification of MuRF1. Data are expressed as means 
$531 \pm \mathrm{SD}(\mathrm{n}=4)$ fold changes relative to CON. One-way ANOVA with Dunnett's post-hoc test,

$532 * P<0.05, * * P<0.01, * * * * P<0.0001$ compared to $\mathrm{CON}$.

\section{Figure 4. A dose-response effect of S6K1 inhibitor on Atrogin-1 and MuRF1 protein}

\section{4 contents in C2C12 myotubes.}

$535 \mathrm{C} 2 \mathrm{C} 12$ myotubes were treated with DMSO $(0.1 \%, 3 \mathrm{~h})$ as a vehicle control $(\mathrm{CON})$ or PF-

5364708671 at the indicated doses for $3 \mathrm{~h}$. Lysates were analyzed by SDS-PAGE and western

537 blotting with the indicated antibodies. (A) Representative images of 2 independent

538 experiments. (B) Quantification of Atrogin-1. (C) Quantification of MuRF1. Data are

539 expressed as means $\pm \mathrm{SD}(\mathrm{n}=4)$ fold changes relative to CON. One-way ANOVA with

540 Dunnett's post-hoc test, $* P<0.05, * * P<0.01, * * * P<0.001$, $* * * * P<0.0001$ compared to

541 CON. (D) Pearson's correlation coefficient to identify the association between p-

$542 \mathrm{rpS6} 6^{\mathrm{Ser} 240 / 244} / \mathrm{rpS} 6$ and Atrogin-1 or MuRF1.

\section{Figure 5. A time course effect of S6K1 inhibitor on Atrogin-1 and MuRF1 protein}

\section{4 contents in C2C12 myotubes.}

$545 \mathrm{C} 2 \mathrm{C} 12$ myotubes were treated with $\operatorname{DMSO}(0.1 \%, 24 \mathrm{~h})$ as a vehicle control $(\mathrm{CON})$ or PF-

$5464708671(30 \mu \mathrm{M})$ for up to $24 \mathrm{~h}$. Lysates were analyzed by SDS-PAGE and western blotting

547 with the indicated antibodies. (A) Representative images from one of two experiments. (B)

548 Quantification of Atrogin-1. (C) Quantification of MuRF1. Data are expressed as means \pm

$549 \mathrm{SD}(\mathrm{n}=4)$ fold changes relative to CON. One-way ANOVA with Dunnett's post-hoc test, ${ }^{*} P$

$550<0.05, * * P<0.01, * * * P<0.001$ compared to CON.

551 Figure 6. Atrogin-1 and MuRF1 protein contents are differentially regulated in Akt and

552 the rapamycin-sensitive mTOR-S6K1 signaling pathway.

553 Insulin/IGF-1/Akt/FoxO signaling pathway is a predominant mechanism regulating Atrogin-

5541 and MuRF1 expression at both mRNA transcription and protein levels in skeletal muscle. 
555 Upon insulin or IGF-1 stimulation, the binding of their respective receptors triggers a

556 signaling cascade to activate Akt. Akt phosphorylates and inhibits FoxO by preventing their

557 localization to the nuclei, and thus FoxO remains in the cytoplasm. In catabolic conditions,

558 FoxO is less phosphorylated and remains in the nuclei to promote Atrogin-1 and MuRF1

559 mRNA transcription and thereby increasing their protein content. Inhibition of mTORC1 or

560 S6K1, one of the Akt downstream signaling, can promote Atrogin-1, but not MuRF1, protein

561 content without altering Akt and FoxO phosphorylation. The evidence indicates that Atrogin-

5621 and MuRF1 protein content are regulated by at least two different mechanisms. How

563 rapamycin-sensitive mTOR and S6K dependent signaling pathway regulate Atrogin-1 protein

564 content remains undetermined. 

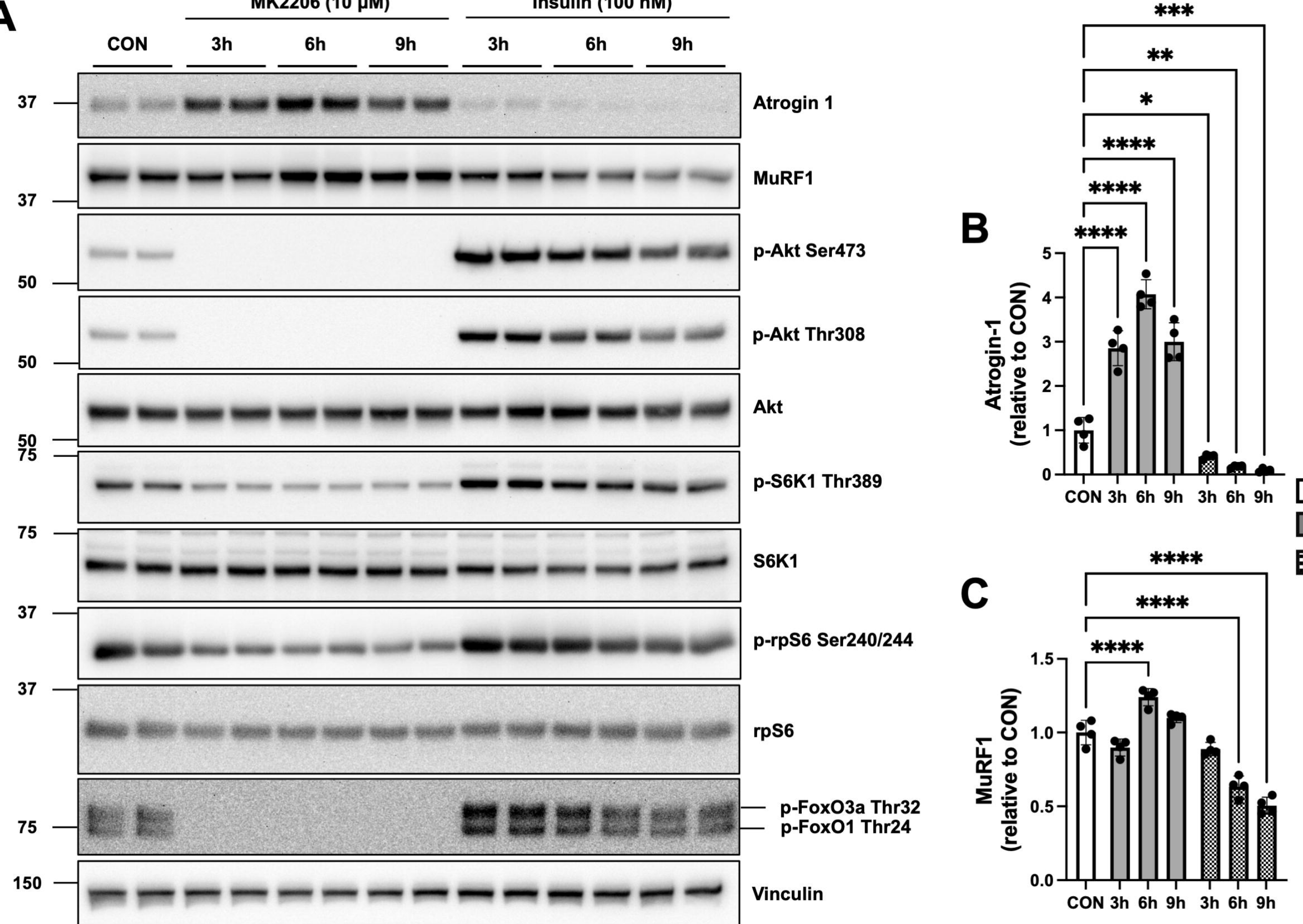

$\square$ CON (DMSO, $\mathrm{H}_{2} \mathrm{O}-0.1 \%$, 9h) $\square$ MK2206 (10 $\mu \mathrm{M})$ \&a Insulin (100 nM)

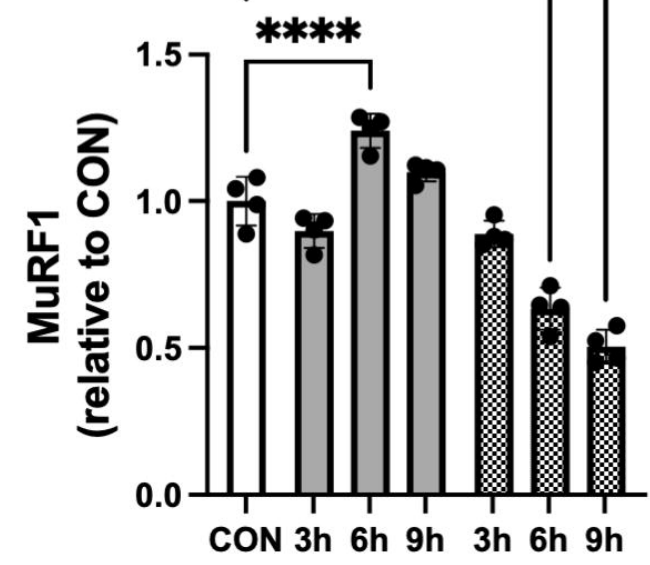


B

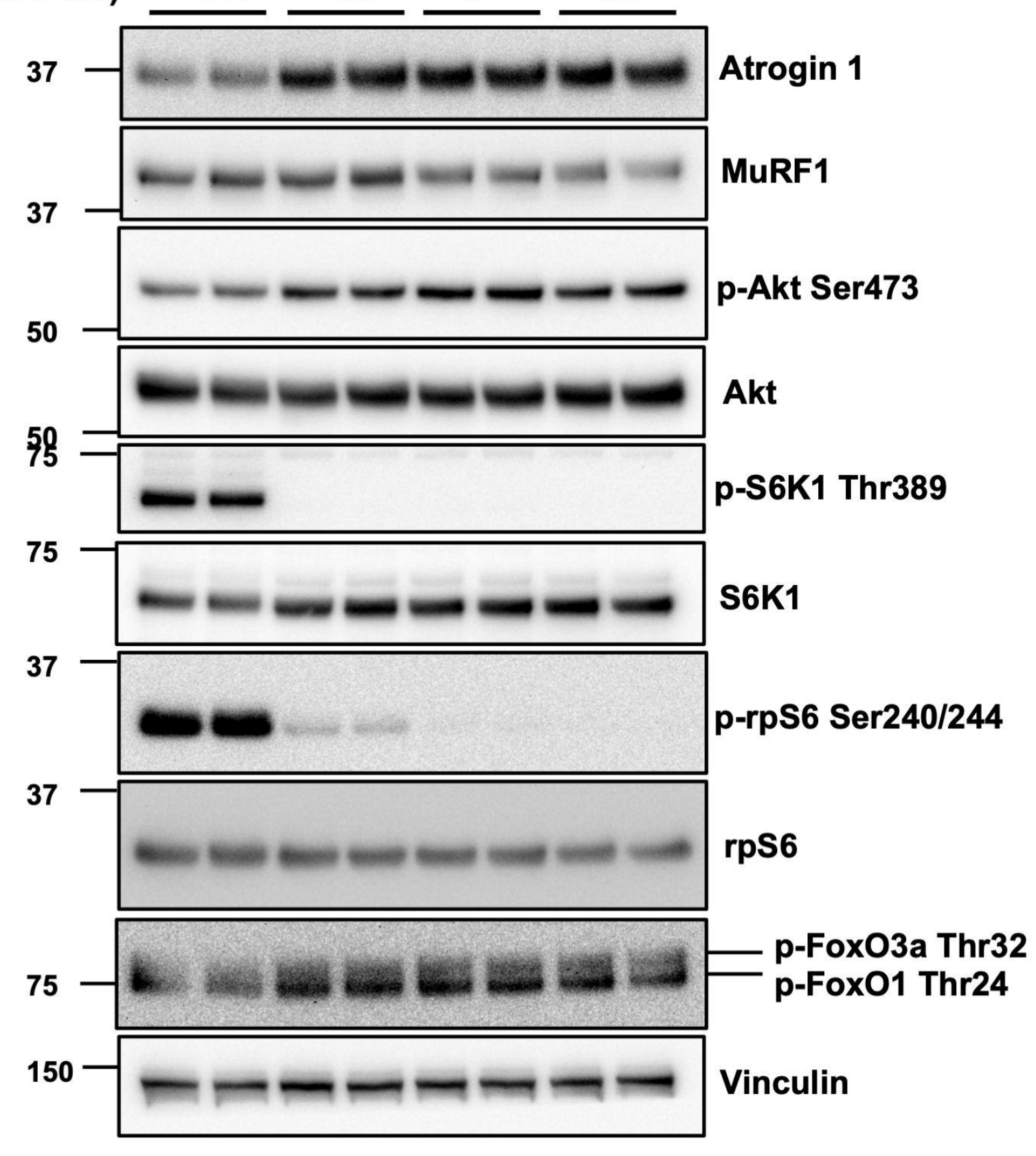

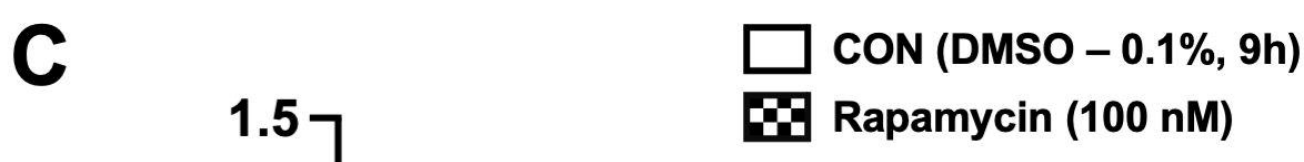

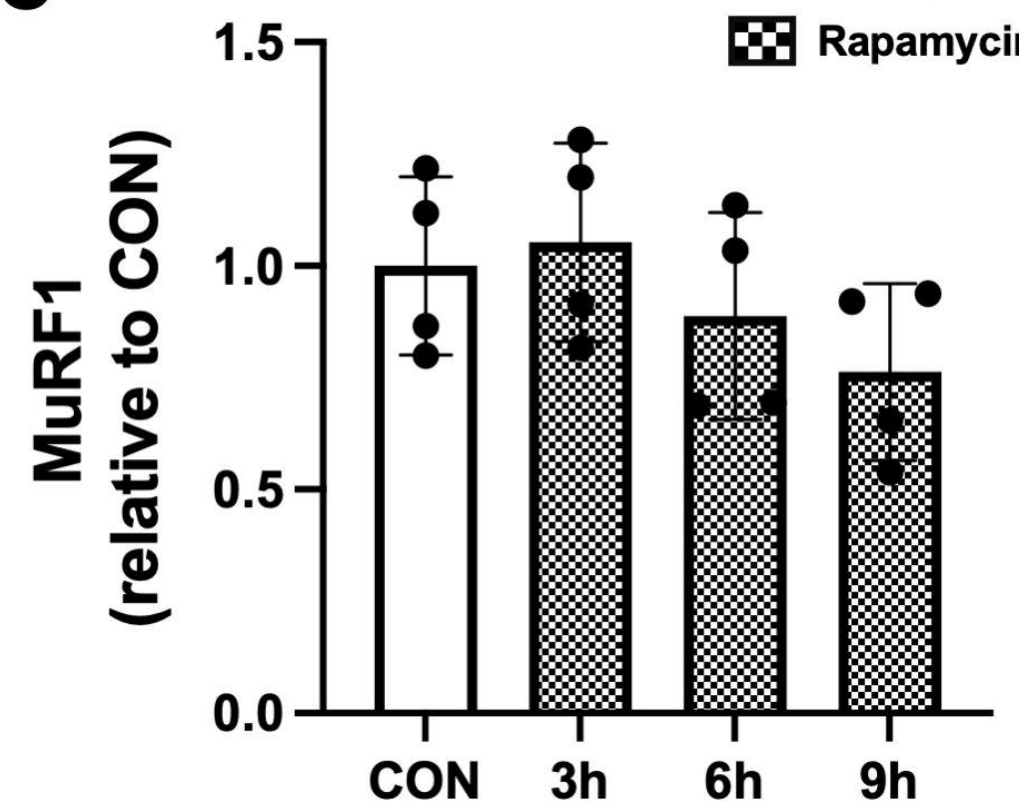


$991(20 \mu \mathrm{M}) \stackrel{\text { CON }}{3 \mathrm{~h}} \stackrel{6 \mathrm{~h}}{\underline{9 h}}$

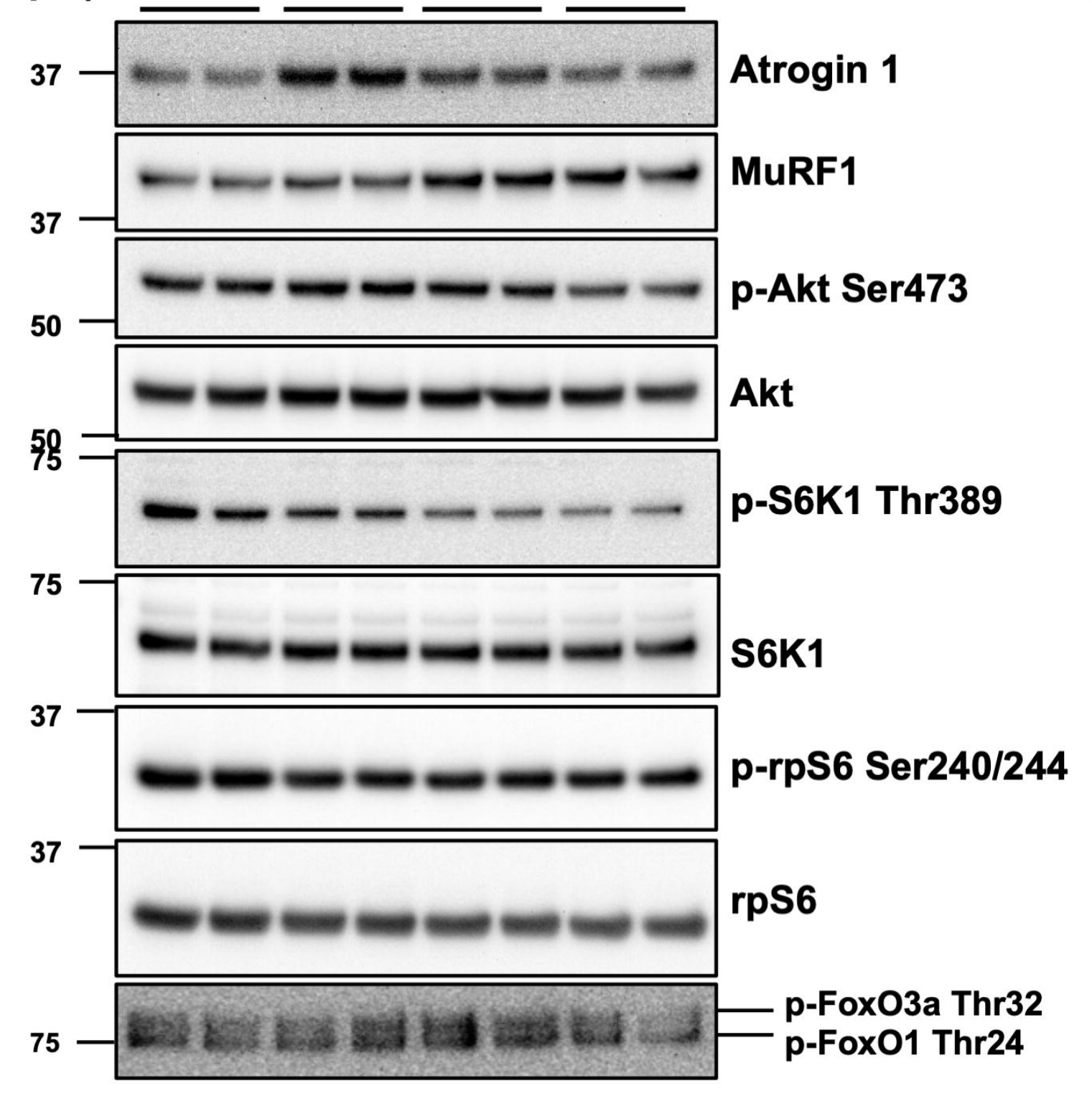

$991(20 \mu \mathrm{M})$ CON $3 \mathrm{~h} 6 \mathrm{~h} 9 \mathrm{~h}$

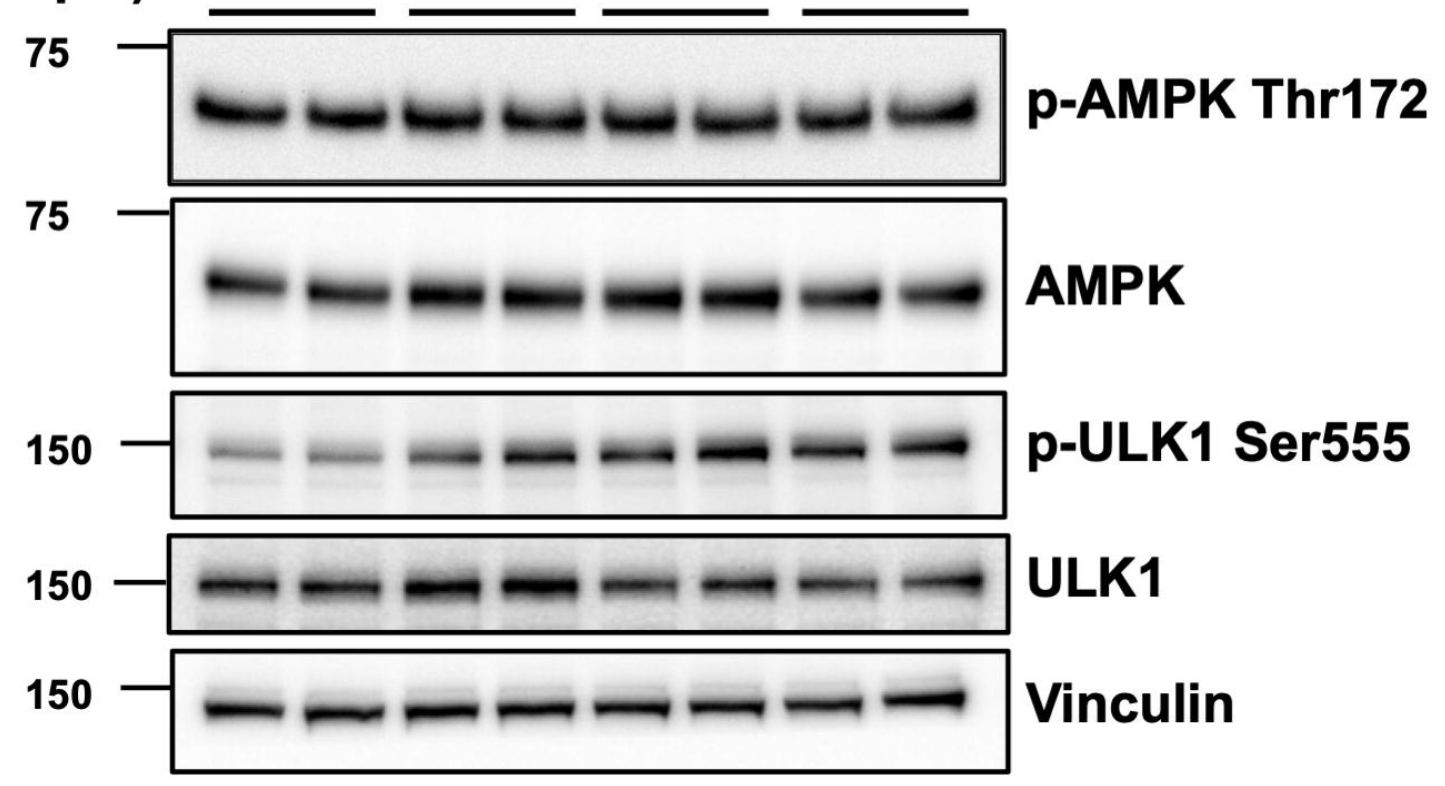

$\square$ CON (DMSO - 0.1\%, 9h)

B

B $991(20 \mu \mathrm{M})$

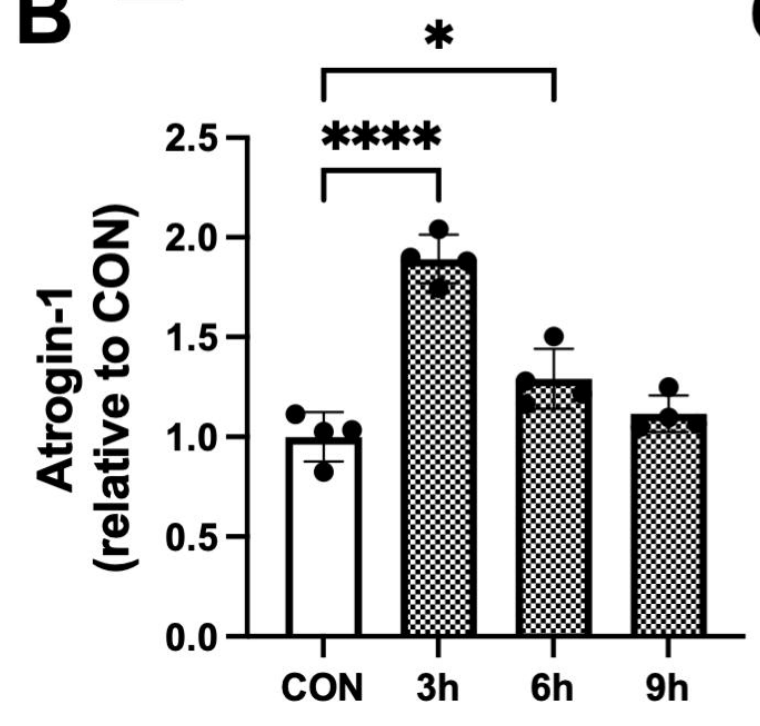

C

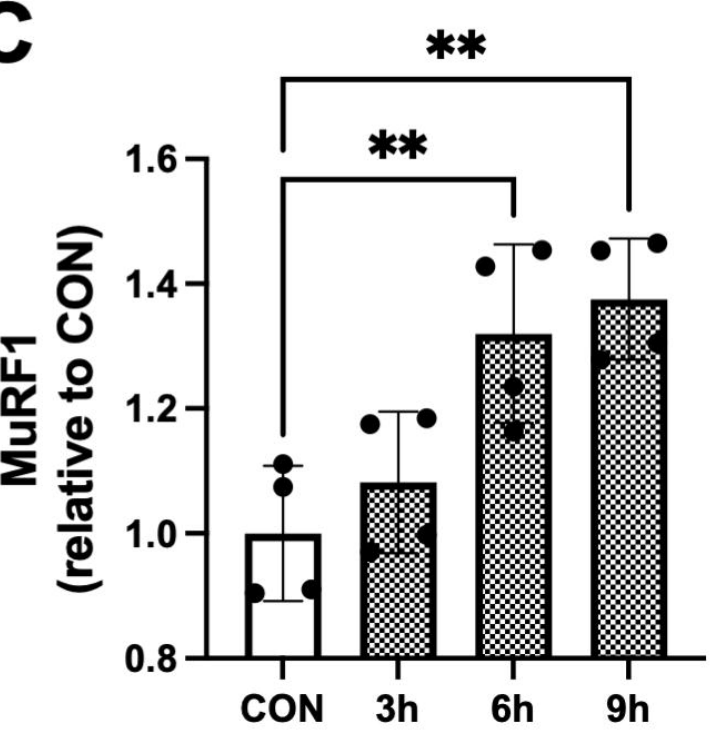


PF-4708671 (3h) CON $10 \mu \mathrm{M} 20 \mu \mathrm{M} 30 \mu \mathrm{M} 40 \mu \mathrm{M} 50 \mu \mathrm{M}$ A

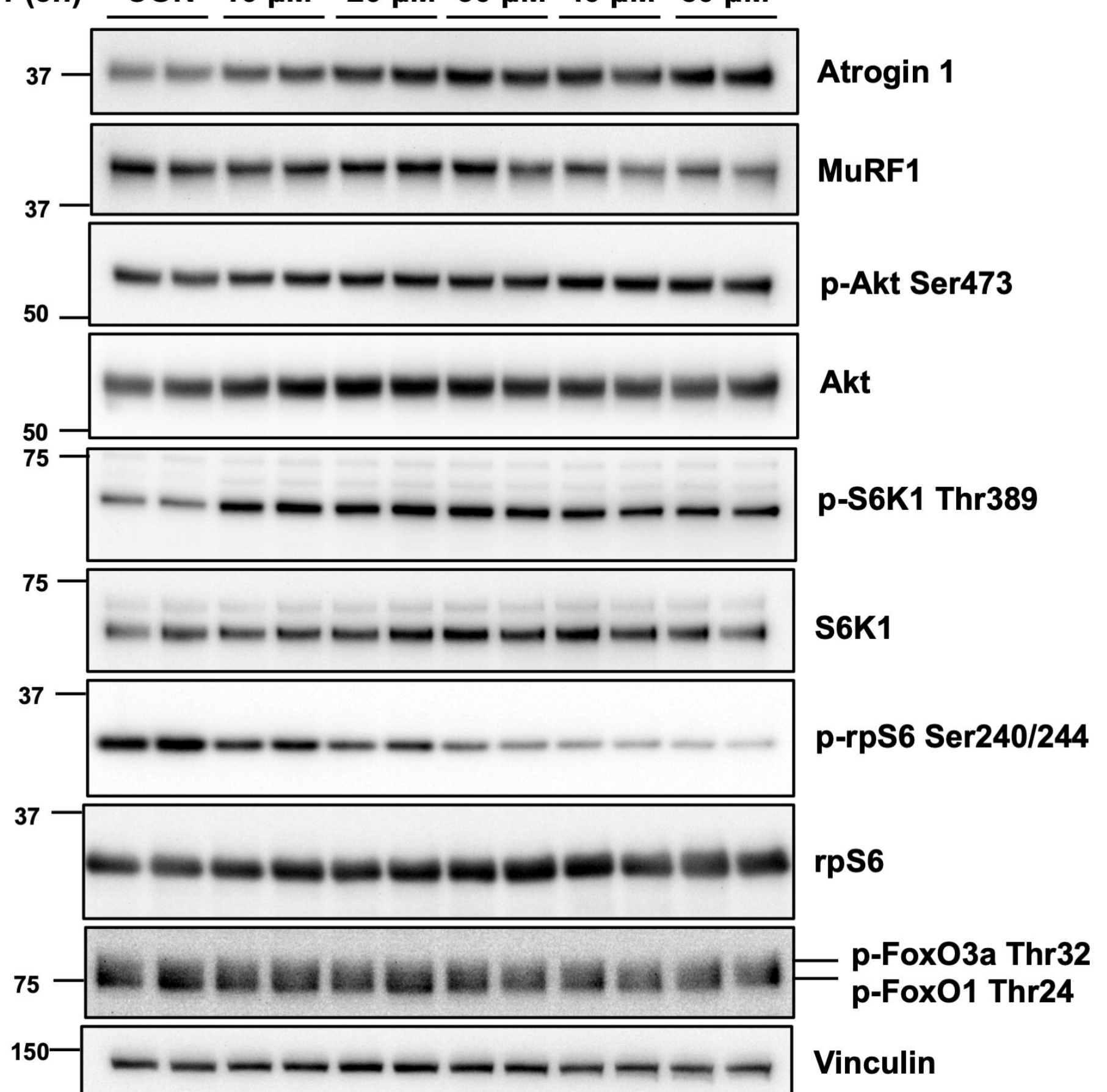

B

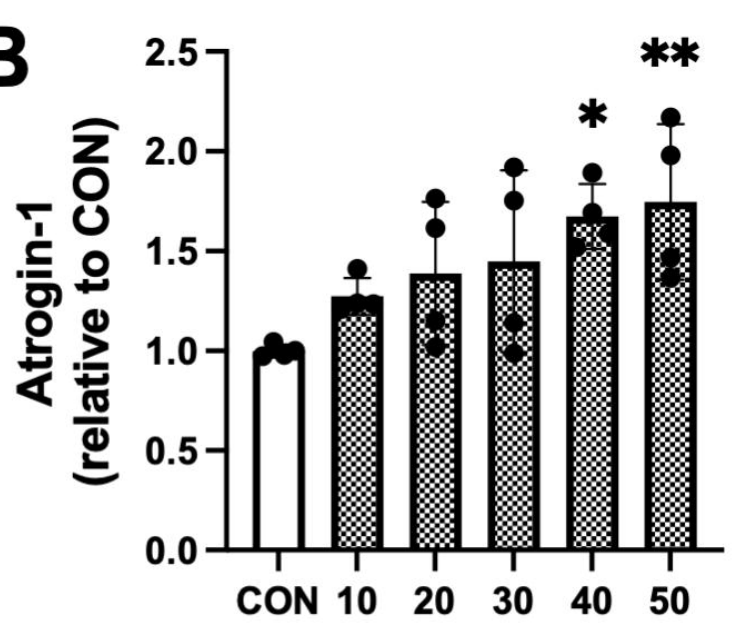

C

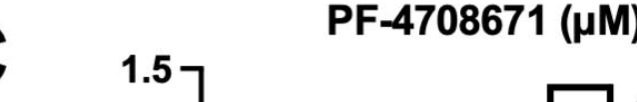

D

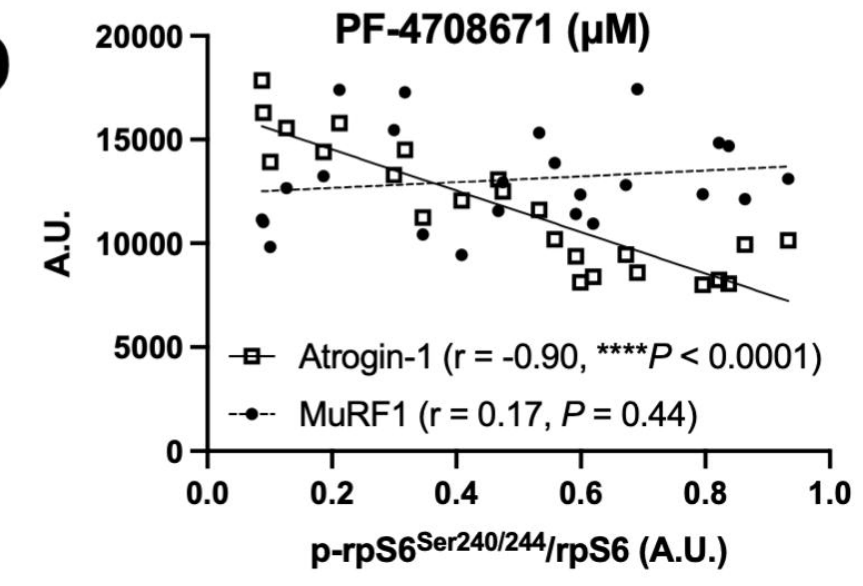


PF-4708671 (30 $\mu \mathrm{M}) \quad$ CON $1 \mathrm{~h} \quad 3 \mathrm{~h} \quad 6 \mathrm{~h} \quad 9 \mathrm{~h} \quad 24 \mathrm{~h}$

A

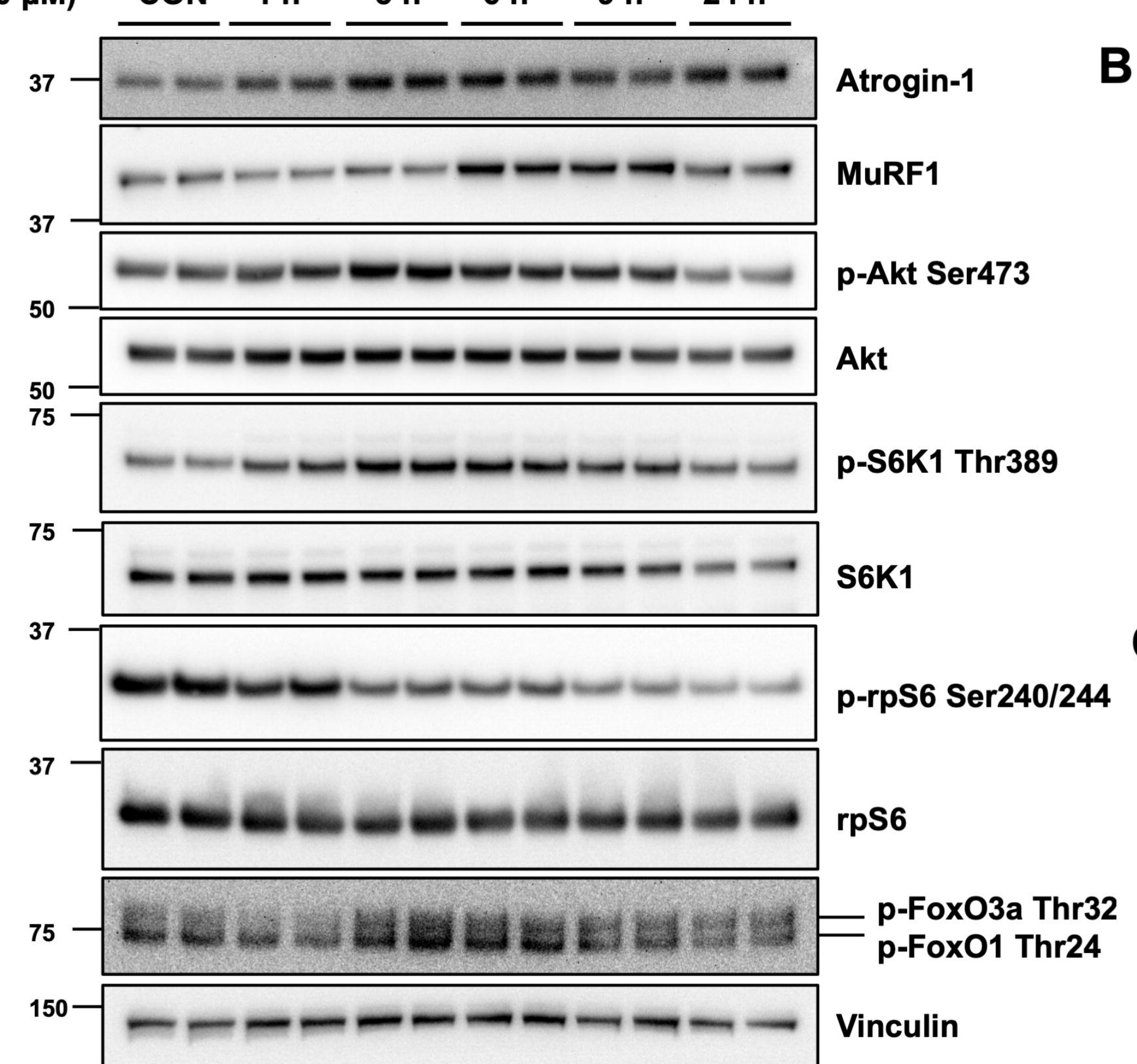

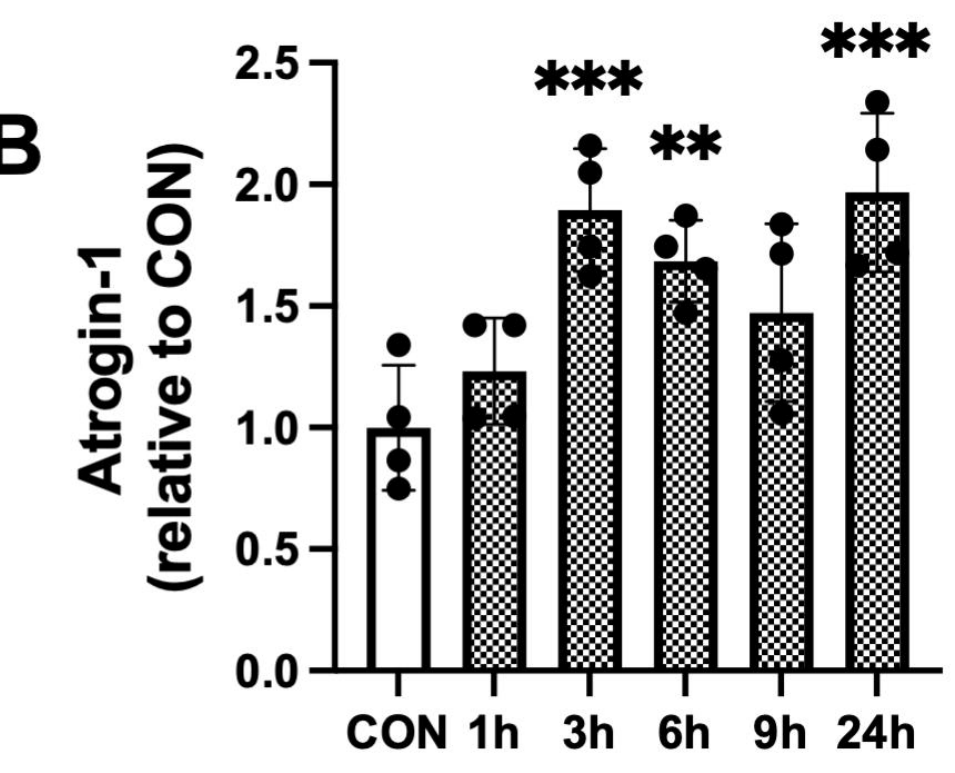

$\square \operatorname{CON}$ (DMSO - 0.1\%, 24h) D PF-4708671 (30 $\mu \mathrm{M})$

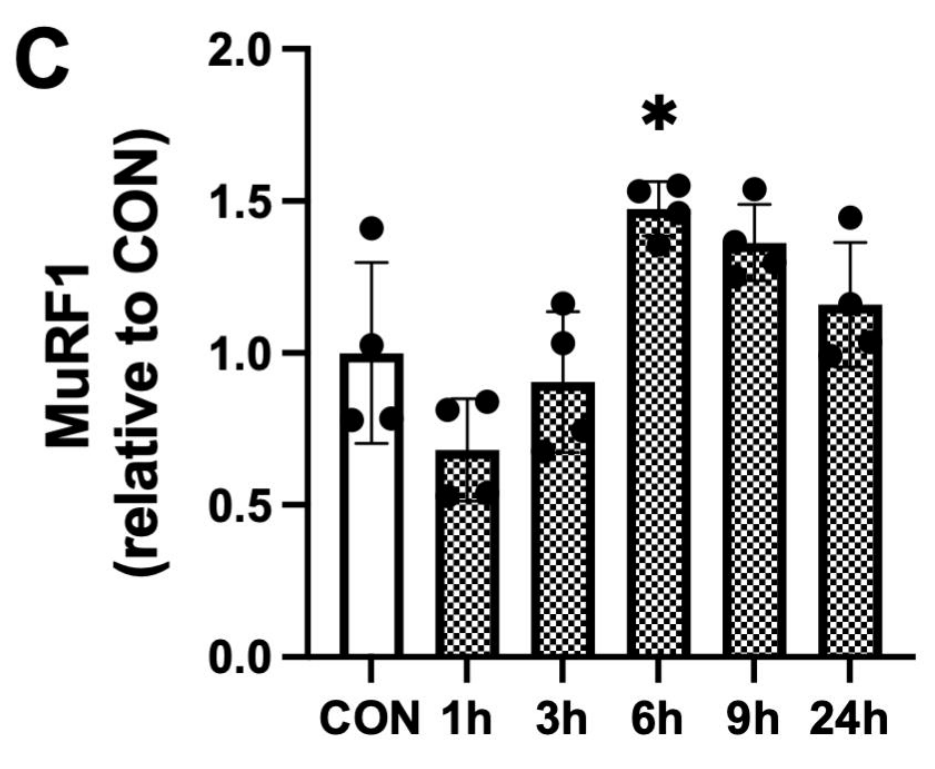




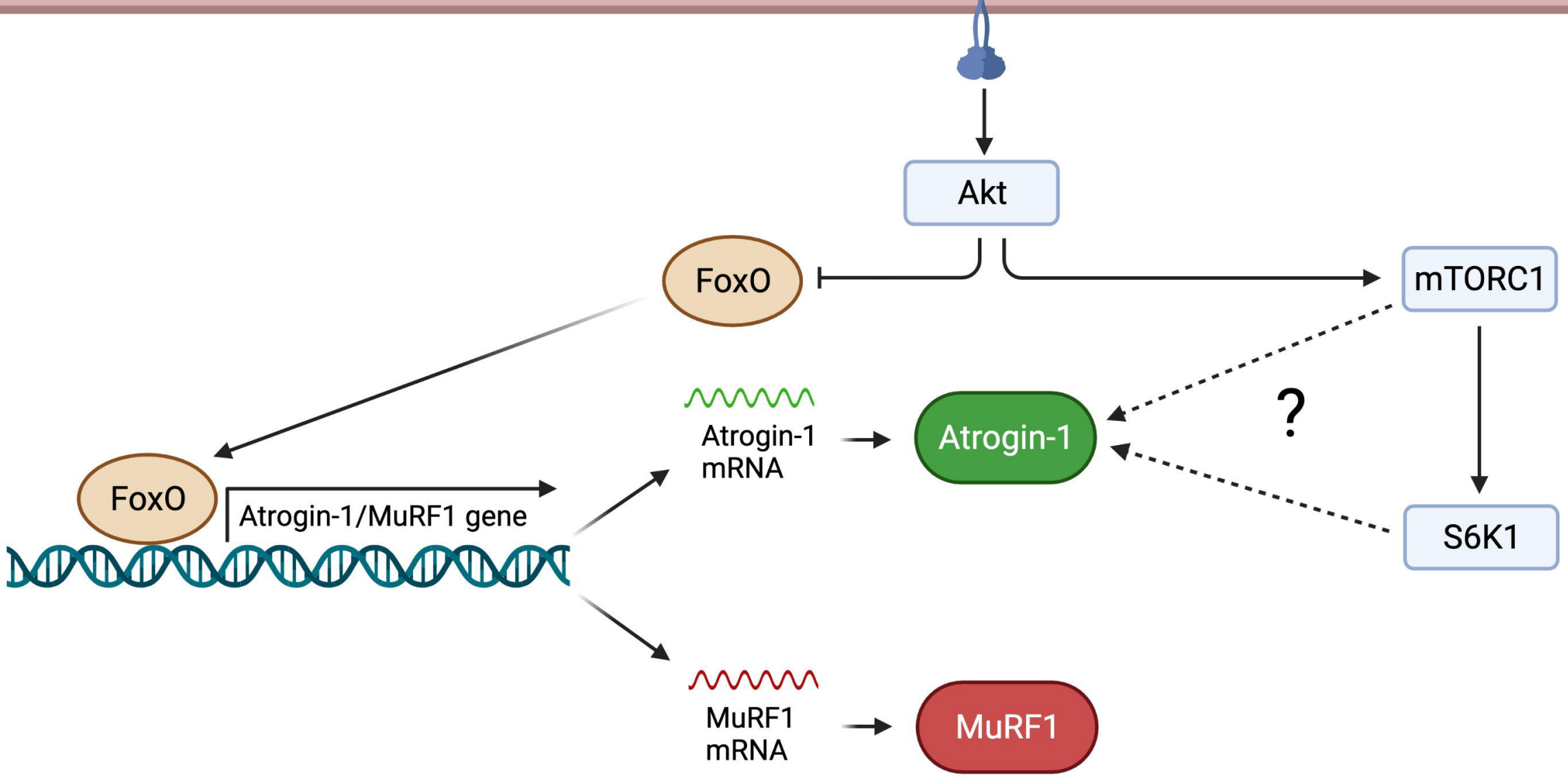

